\title{
Sustainable Urban Community Eco-Feedback through Simulation-enabled Performance Dashboards
}

\author{
Tarek Rakha ${ }^{1}$, Elena Echarri ${ }^{2}$, Elizabeth Krietemeyer ${ }^{2}$ and Jason Dedrick ${ }^{3}$ \\ ${ }^{1}$ School of Architecture, Georgia Institute of Technology, Atlanta, GA, USA \\ ${ }^{2}$ School of Architecture, Syracuse University, Syracuse, NY, USA \\ ${ }^{3}$ School of Information Studies, Syracuse University, Syracuse, NY, USA
}

\begin{abstract}
As global energy consumption is on the rise, energy usage awareness technologies are needed to reduce negative environmental effects. Energy monitoring technologies, known as Eco-Feedback Systems, are attempting to serve as tools that motivate individuals and communities to adopt sustainable living behaviours. However, their feedback to users is limited to past and present measured data, with limited ability to predict future energy consumption when users adopt more sustainable practices. This paper, therefore, focuses on developing a workflow for an interactive dashboard interface that allows the user to track and make energy-conscious decisions. The workflow uses calibrated urban-scale simulation-based predictions of future residential energy use data, to provide real-time tailored display feedback of various energy behaviour and design alteration scenarios.
\end{abstract}

\section{Introduction}

Climate change awareness continues to be a concern, as technologies are further developed to measure and monitor the world (Pittock, 2005). In the United States, households account for about $38 \%$ of national carbon emissions and are the second largest sector of energy consumption (Pachauri, 2014). Such data confirms that residential energy use is the source of one of the largest leaps towards the "feared" climate change phenomenon (Fleming, 1998). In order to mitigate climate change effects, researchers are attempting to create public awareness about this threat, especially within homes, through new "Eco-feedback" technologies, (Horn et al., 2011, Karlin et al. 2014) which are defined as "any technology that provides feedback on individual or group behaviours with a goal of reducing environmental impact" (Froehlich, Findlater, and Landay, 2010).

An earlier investigation asked a total of 505 participants to report their perceptions about individual-scale energy consumption. The study demonstrated that most surveyed householders are unable to accurately estimate their energy usage. These results imply that in order to promote sustainable attitudes within home stakeholders, the lack of public knowledge about energy consumption and proenvironmental behaviours must be addressed. (Attari, DeKay, Davidson, and De Bruin, 2010). Other research suggests that with education about specific energy-based actions and behaviours, households could reduce annual energy consumption by almost $30 \%$, which is approximately $11 \%$ of the total US energy use (Gardner and Stern, 2008), as well as a potential reduction of $20 \%$ $\mathrm{CO} 2$ emissions over the next 10 years (Jain, Taylor, and Peschiera, 2012). These findings motivate the exploration of human reaction to information about more sustainable household practices, in order to develop better educational tools that promote energy efficient behaviours. Gardner and Stern's research revealed that most residents are motivated to take pro-environmental action, but do not have the knowledge to do so. Traditional tools such as internet-publicized lists that provide advice on energy-savings actions are often not accurate, and conventional gadgets that measure real-time energy usage have not demonstrated a significant impact on users. Further research shows that the majority of traditional energy monitors such as Green Button web sites provide aggregated numerical data in the form of single daily or weekly values which do not allow the user to identify and correct behavioural mistakes from previous days (Constanza, Ramchurn, and Jennings, 2012), and therefore cannot be considered as useful tools to unfamiliar users.

Interactive systems of Eco-feedback--unlike noninteractive modes of energy visualization--can be used to better advise users on their daily energy-use activities, and motivate them to adopt a more sustainable lifestyle (Pierce, Odom, and Blevis, 2008). Results from a survey conducted with twelve participants to test a new interactive system of Eco-feedback known as "FigureEnergy" for a period of two weeks demonstrated that even among users with previous experience with Ecovisualization systems, knowledge on appliance-level energy use increased significantly. This suggests that results from interactive Eco-feedback may be more successful than traditional representation systems (Constanza, Ramchurn, and Jennings, 2012).

There is an opportunity to develop new systems of user engagement that provide accurate, real-time estimations of future energy use and $\mathrm{CO} 2$ emissions based on present energy-efficient behaviours and design retrofits. The challenges associated with limited accuracy and user motivation presented by current Eco-feedback displays have created a demand for smart interfaces that provide the user with constantly-updated and engaging results, thus allowing for personal decisions regarding future energy use. The difficulties presented by the data representation of most Eco-visualization and feedback systems, such as untailored advice, incomprehensible 
metrics and highly aggregated representations, become an obstacle for further improvement. Previous work suggests that simulation-based data may be an effective solution to resolve such issues, serving as a basis to provide more accurate, comprehensible and actionable feedback. An investigation conducted in the City of Osaka, Japan, which utilized a District Clustering model approach to simulate different future $\mathrm{CO} 2$ emission scenarios based on environmental changes on individual, collective and policy behaviours demonstrated that simulations and speculative data analysis could lead to the development of energy-saving strategies capable of reducing the total $\mathrm{CO} 2$ emissions of Osaka by $80 \%$ (Yamaguchi, Shimoda, and Mizuno, 2007).

By using simulation tools, predictions of experimental conditions and their respective performance become possible, even prior to the conditions even existing (Hensen, 1992). Through specific iterative design and analysis methods, simulations of potential future conditions allow for testing the best-performing scenarios, and therefore, determining the most desirable outcomes (Petersen \& Svendsen, 2010). In this paper, we speculate on combining simulation with an engaging design of an interactive user-friendly energy dashboard to support sustained user engagement and behavioural change. By allowing the user to select the behavioural changes of interest, rather than provide a generic list, and by providing the respective future savings linked to such actions, motivation to implement such tools of feedback into everyday routines may be more effective and easier, and users would feel more motivated to reduce consumption.

\section{Methodology}

In order to develop a simulation-based Eco-feedback platform and successfully investigate its effects on both individual and community total energy consumption, an Urban Building Energy Model (UBEM) is first built and calibrated to simulate existing human behaviours and consequent energy performance based on real-life energy measures. The results of a calibrated model are then validated for analysis and can be utilized as informative and speculative content for the interface. The results of a case-study run in a residential community in Austin, Texas, USA that utilizes appliance-level eGauge and smart meter data are used as validation. The research method presented in this section follows a threefold structure: 1) pre-processing of measured data and functional clustering workflows for model calibration; 2) developing the UBEM for an existing community, and simulating behaviour change and design scenarios; and 3) data visualization for comparative analysis and ecofeedback dashboard integration. A more detailed overview of the method is presented in Figure 1.

\section{Data Pre-processing and Calibration}

The processing and calibration of measured data is necessary for the successful development of a simulationbased comparative model. To ensure the veracity of simulated results from the current neighbourhood energy usage, data collected from appliance-level eGauge meters that measure energy use in $\mathrm{kWh}$ for all building equipment were used to calibrate the inputs for the UBEM. In the presented methodology, the analysis and sorting of measured data for the development of the model is performed utilizing a data clustering and schedule profiling method previously proposed by part of the research team (El Kontar and Rakha, 2019). Firstly, recorded energy-use data was accessed and organized into hourly datasets for annual time periods. These are then arranged by building unit and divided based on energy load type (cooling, heating, lighting and equipment), time in months and energy unit type. Any missing or corrupted data is cleared to avoid potential clustering errors. After data pre-processing, a functional clustering analysis (Bouveyron et al., 2015) is performed on the hourly energy data, and the optimal number of energy cluster types is selected utilizing a Bayesian Information Criterion (BIC) (Shwartz, 1978). The measured hourly

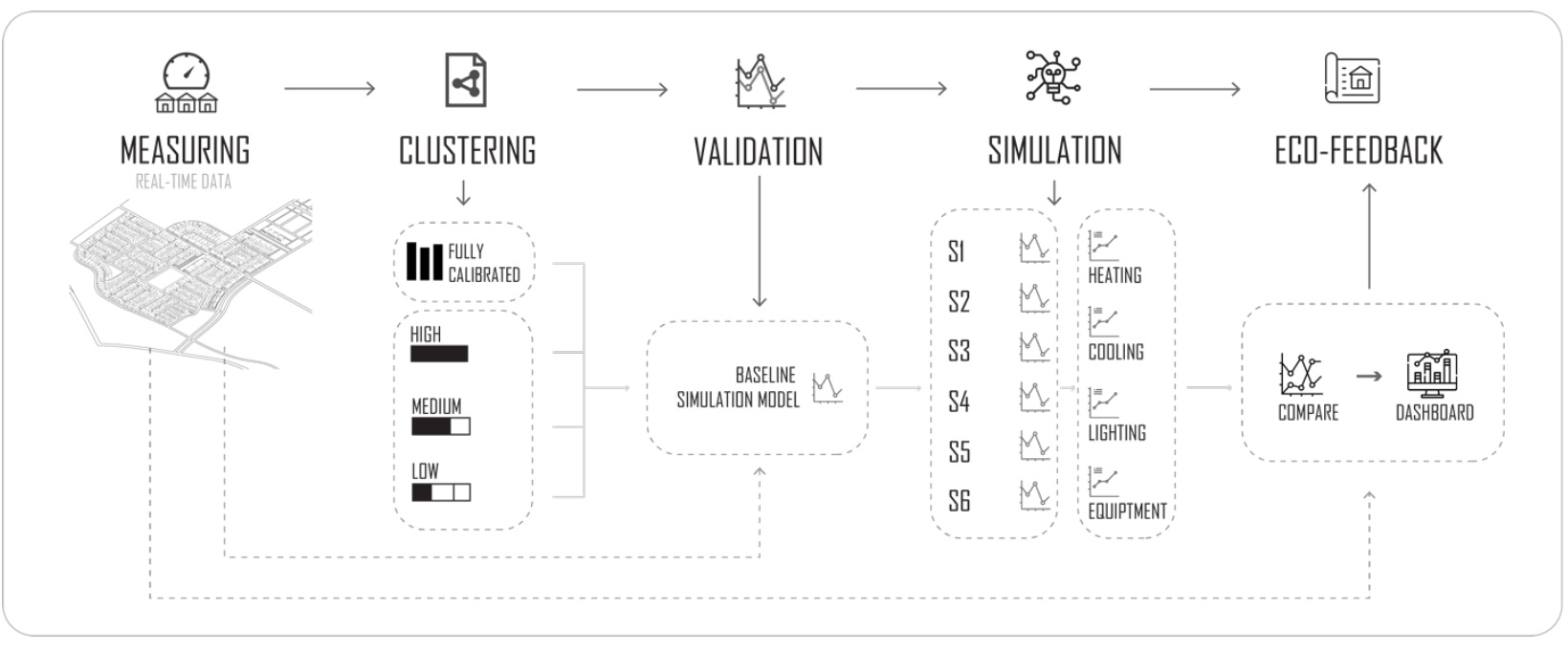

Figure 1: Research method, showcasing the integration of a validated UBEM for Eco-feedback displays. 


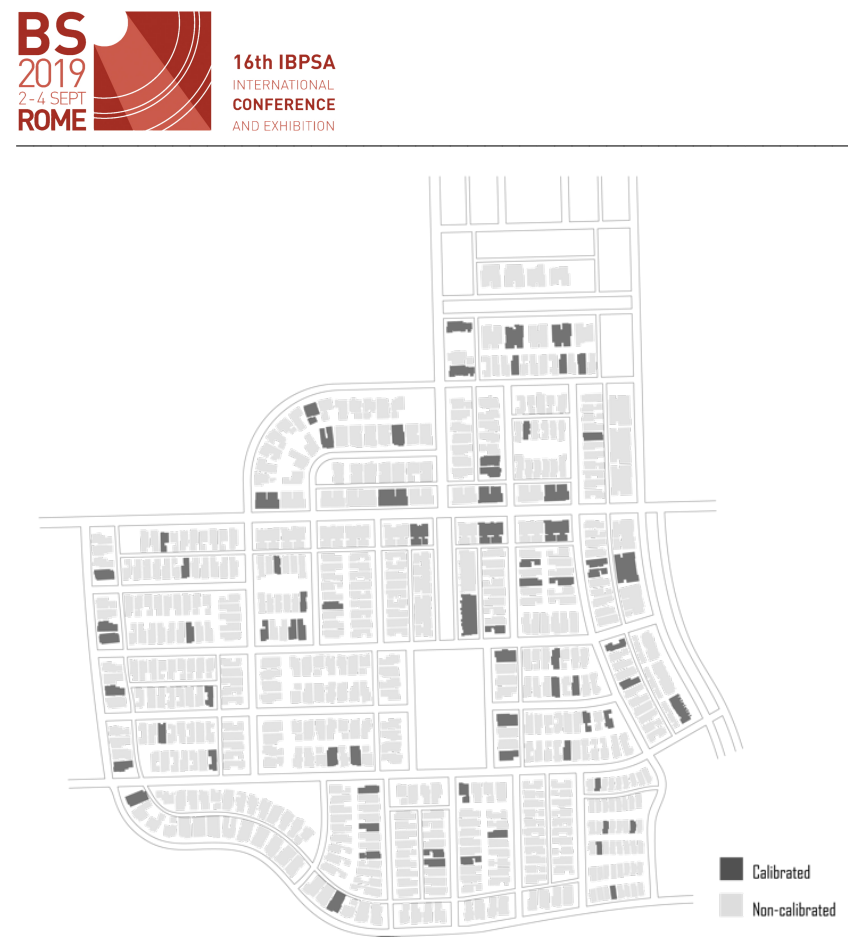

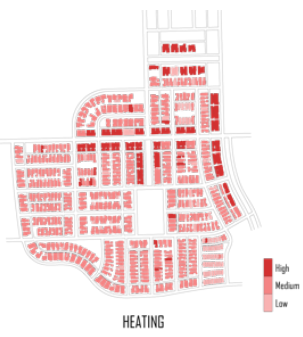
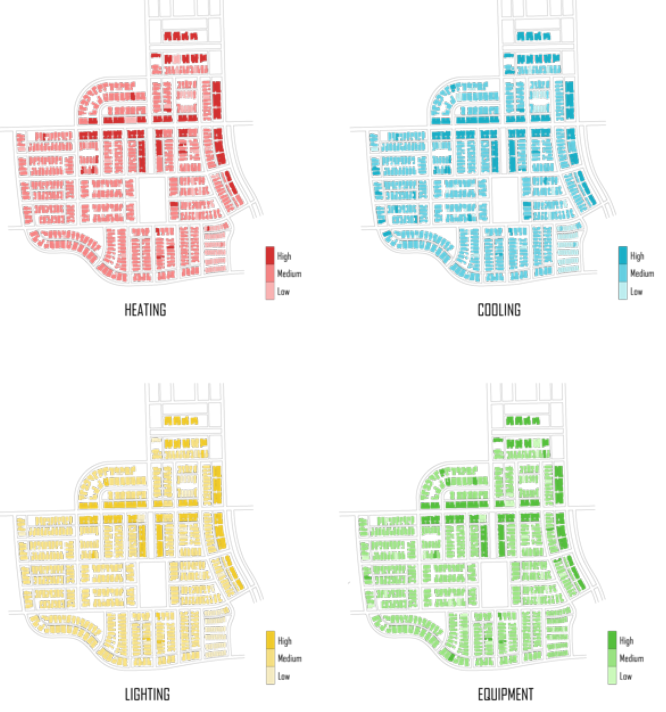

Figure 2: Calibrated and semi-calibrated UBEM components, with designation of high, mid and low users.

data for each building is then compiled and plotted into daily mean profiles, visually compared and grouped according to their closest energy consumption cluster group. Based on energy consumption values, buildings in this study are grouped into three different cluster types: High energy consumption, Medium energy consumption and Low energy consumption buildings.

\section{UBEM Development and Scenario Simulation}

Once processed, resultant clustering analysis profiles are translated into energy usage schedules and intensities. The translation of behavioural energy use profiles is achieved using the formula below.

$$
F_{c}\left(t_{1}, t_{2}\right)=\int_{t_{1}}^{t_{2}} y_{v c}(t) \cdot d t / \text { Max Load }
$$

Where $F_{c}\left(t_{1}, t_{2}\right)$ is the fraction to be applied to a load between time instances $t_{1}$ and $t_{2}$ for a specific variable cluster $v c$, where $v \in\{l, e, c, h\}$ where $1, \mathrm{e}, \mathrm{c}, \mathrm{h}$ are lighting, equipment, cooling, and heating respectively. Calibrated data is used to analytically deduce usage and behaviour daily schedules. A building simulation daily occupancy schedule is a table of values ranging from 0 to 1 , with a value less than 1 indicating that only a fraction of all possible occupants are present. Each entry in the table corresponds to a simulation time step. For example, if the time step is one hour long, there will be 24 schedule entries. The value of the schedule at time step $t$ indicates the portion of the maximum normal building occupancy present at time $t$. For each cluster type, a different energy usage schedule is generated for the 24 hours of daily simulation in accordance with the behaviour of their respective occupants and the energy consumption intensity. These schedules are then combined with inputs for cooling, heating, lighting and equipment appliances accordingly and assigned to the buildings in each respective cluster type. Buildings whose energy data is missing or has been corrupted are assigned one of the three cluster types according to estimations based on their size and location within the neighbourhood, while buildings which contain all data are assigned the schedules determined by the clustering analysis results. Individual building specifications for both calibrated and semi-calibrated buildings are generated as templates, and combined into a baseline template library in the Urban Modelling Interface (UMI) plug-in for Rhino3D as a front-end (Reinhart et al, 2013). Energy+ is the simulation engine (Crawley et al, 2001) in which each building template is assigned to its respective building geometry on a three-dimensional massing model, completing the UBEM generation process. In order to successfully create the UBEM model, UMI data inputs for weather information, material properties, construction and geometry specifications, as well as parameters for conditioning and energy loads were specified to match measured data. It is important to note that the simulation engine has some inherent limitations, such as limited capability of simulating long-wave radiation exchange between buildings.

\section{Comparative Eco-Feedback}

The comparison of results was implemented with the extraction of simulated data and visualized graphically. The visualization of schemes for an Eco-feedback dashboard and the integration of the plotted comparative data for design case scenarios is mocked-up. Visualizations for heating, cooling, equipment and total energy loads, as well as timeline comparisons between individually measured and simulated data or with the community can also be represented in the dashboard framework. 


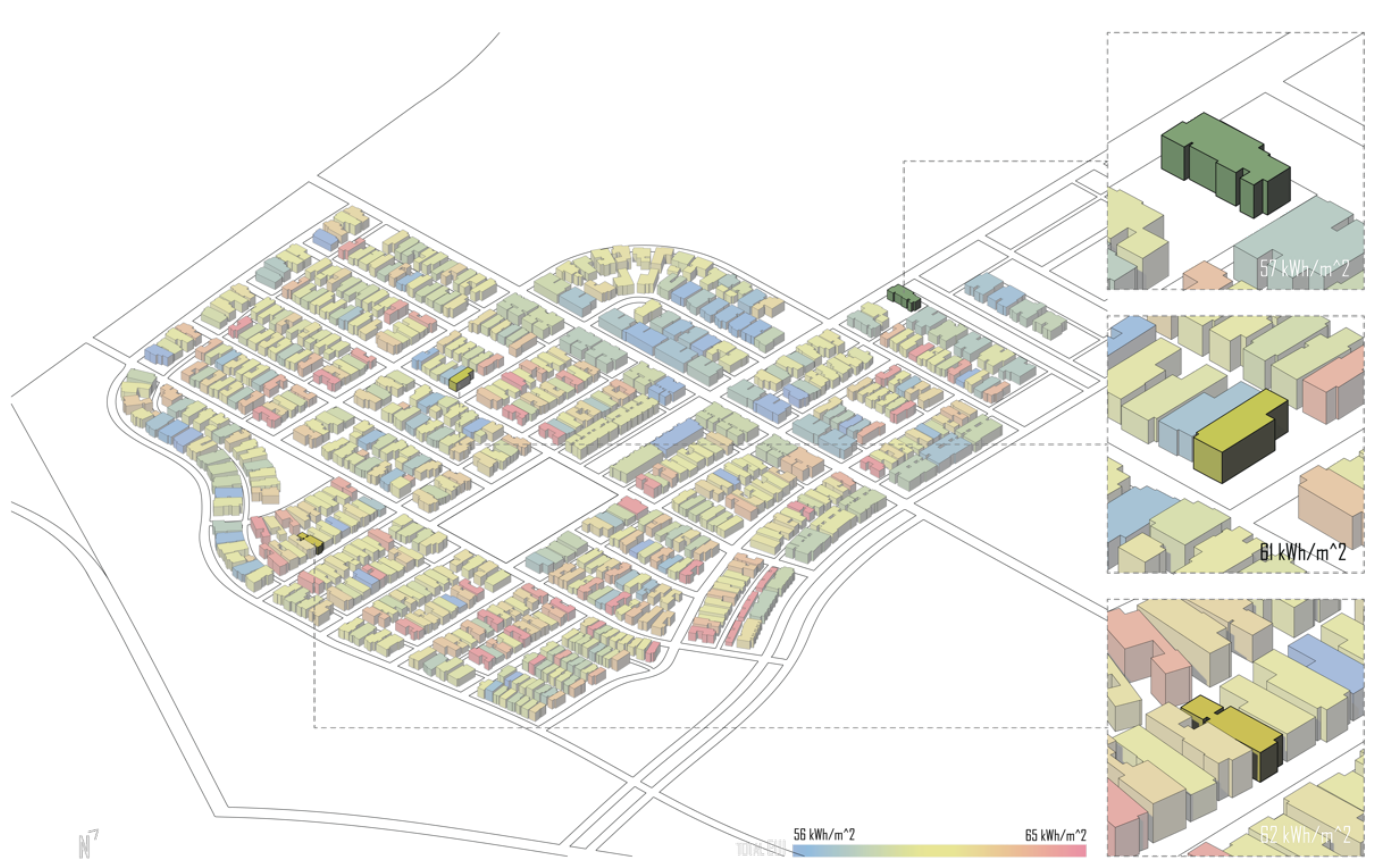

Figure 3: Calibrated UBEM EUI and three buildings selected as high, mid and low users for simulation scenarios.

Table 1: Single and combined action scenarios for three buildings within the calibrated UBEM.

\begin{tabular}{|c|c|c|c|c|c|c|c|c|c|c|}
\hline & $\begin{array}{c}\text { B } \\
\text { Baseline }\end{array}$ & $\begin{array}{c}\text { S1 } \\
\text { Lower } \\
\text { Setpoints }\end{array}$ & $\begin{array}{c}\text { S2 } \\
\text { Lower } \\
\text { Max. } \\
\text { Capacity }\end{array}$ & $\begin{array}{c}\text { S3 } \\
\text { Heat } \\
\text { Recov. }\end{array}$ & $\begin{array}{c}\text { S4 } \\
\underset{g}{\text { Dimmin }}\end{array}$ & $\begin{array}{c}\text { S5 } \\
\text { Low } \\
\text { Lighting } \\
\text { Power }\end{array}$ & $\begin{array}{c}\text { S6 } \\
\text { Interior } \\
\text { Shading }\end{array}$ & $\begin{array}{c}\text { S7 } \\
\text { Low } \\
\text { Equip. } \\
\text { Power }\end{array}$ & $\begin{array}{c}\text { S8 } \\
\text { Natural } \\
\text { Vent. }\end{array}$ & $\begin{array}{c}\text { S9 } \\
\text { Daytime } \\
\text { Lights } \\
\text { Off }\end{array}$ \\
\hline \multicolumn{11}{|l|}{ Heating } \\
\hline Setpoint $\left({ }^{\circ} \mathrm{C}\right)$ & 19 & 17 & 19 & 19 & 19 & 19 & 19 & 19 & 19 & 19 \\
\hline Max Capacity $\left(\mathrm{W} / \mathrm{m}^{2}\right)$ & 100 & 80 & 100 & 100 & 100 & 100 & 100 & 100 & 100 & 100 \\
\hline \multicolumn{11}{|l|}{ Cooling } \\
\hline Setpoint $\left({ }^{\circ} \mathrm{C}\right)$ & 26 & 28 & 26 & 26 & 26 & 26 & 26 & 26 & 26 & 26 \\
\hline Max capacity $\left(\mathrm{W} / \mathrm{m}^{2}\right)$ & 100 & 80 & 100 & 100 & 100 & 100 & 100 & 100 & 100 & 100 \\
\hline Heat Recovery & - & - & Yes & - & - & - & - & - & - & - \\
\hline \multicolumn{11}{|l|}{ Lighting } \\
\hline Power Density $\left(\mathrm{W} / \mathrm{m}^{2}\right)$ & 1 & 1 & 1 & 1 & 1 & 0.5 & 1 & 1 & 1 & 1 \\
\hline Desired Illuminance & 150 & 150 & 150 & 150 & 100 & 150 & 150 & 150 & 150 & 150 \\
\hline Dimming & - & - & - & - & Yes & - & - & - & - & - \\
\hline Interior Shading & - & - & - & - & - & - & Yes & - & - & - \\
\hline \multicolumn{11}{|l|}{ Equipment } \\
\hline Power Density $\left(\mathrm{W} / \mathrm{m}^{2}\right)$ & 3 & 3 & 3 & 3 & 3 & 3 & 3 & 1 & 3 & 3 \\
\hline
\end{tabular}

\begin{tabular}{|c|c|c|c|c|}
\hline & $\begin{array}{c}\text { C1 } \\
\text { Low } \\
\text { Setpoint+ } \\
\text { Shading }\end{array}$ & $\begin{array}{c}\text { C2 } \\
\text { Low } \\
\text { Equip.+ } \\
\text { LPD }\end{array}$ & $\begin{array}{c}\text { C3 } \\
\text { LPD + } \\
\text { Daytime } \\
\text { Light Off }\end{array}$ & $\begin{array}{c}\text { C4 } \\
\text { Setpoint+ } \\
\text { Shading+ } \\
\text { LPD+Eq. }\end{array}$ \\
\hline \multicolumn{5}{|l|}{ Heating } \\
\hline Setpoint $\left({ }^{\circ} \mathrm{C}\right)$ & 17 & 19 & 19 & 19 \\
\hline Max Capacity $\left(\mathrm{W} / \mathrm{m}^{2}\right)$ & 100 & 100 & 100 & 100 \\
\hline \multicolumn{5}{|l|}{ Cooling } \\
\hline Setpoint $\left({ }^{\circ} \mathrm{C}\right)$ & 28 & 28 & 26 & 28 \\
\hline Max capacity $\left(\mathrm{W} / \mathrm{m}^{2}\right)$ & 100 & 100 & 100 & 100 \\
\hline Heat Recovery & - & - & - & - \\
\hline \multicolumn{5}{|l|}{ Lighting } \\
\hline Power Density $\left(\mathrm{W} / \mathrm{m}^{2}\right)$ & 1 & 0.5 & 0.5 & 0.5 \\
\hline Desired Illuminance & 150 & 150 & 150 & 150 \\
\hline Dimming & - & - & - & - \\
\hline Interior Shading & Yes & - & - & Yes \\
\hline \multicolumn{5}{|l|}{ Equipment } \\
\hline PceedRensity fW/me ${ }^{2}$ & th IB'PSA & onference & 3 & $\overline{11}$ \\
\hline
\end{tabular}




\section{Case Study}

The paper focuses on a case study community located in the northeast centre of the city of Austin, Texas, USA with a total area of 711 acres. Using historical measured energy data from 2014, and weather files from of Austin's Actual Meteorological Year (AMY), a UBEM was developed and calibrated for simulation scenarios and investigation of performance. The study simulated a total of 608 buildings, from which 49 were fully calibrated using the functional clustering calibration method. Three cluster types were determined as previously stated, based on the buildings' total EUI measurements. Figure 2 shows the placement of all calibrated buildings and documents the location of all cluster type buildings. Heating results were omitted from the analysis due to its limited impact on total annual energy consumption values in the coolingdominated Austin climate. The R-value for façades was $8.55 \mathrm{~m}^{2} \mathrm{~K} / \mathrm{W}$, the roof was $12 \mathrm{~m}^{2} \mathrm{~K} / \mathrm{W}$, and the window U-value was $1.1 \mathrm{~W} / \mathrm{m}^{2} \mathrm{~K}$. The Window-to-Wall ratio was set uniformly to $30 \%$ in all orientations.

To allow for more comparable and focused results, three buildings were selected to simulate various scenarios, each one corresponding to a different energy cluster type and their respective energy use intensity UBEM inputs. Figure 3 shows the location and cluster type of the selected buildings, as well as a display of their total measured annual energy consumption as documented in the historical data. Using a calibrated UBEM, a total of fourteen scenarios (both single action and combinations of up to three actions) were simulated and compared to measured data. Inputs for cooling and heating set points, lighting and equipment power density, desired illuminance values, ventilation and other variations were altered and tested for reductions in energy usage and improvements. Table 1 documents all scenarios and their specific UMI inputs for the UBEM model. These include comfort set points in which the cooling and heating systems are activated by occupants when experienced, as well as maximum energy capacity of the system in $\mathrm{W} / \mathrm{m}^{2}$. Power density for lighting and equipment is used to represent the systems energy normalized by floor area. Desired illumination for performance is also set for electric lighting to automatically turn on if this illumination threshold was not available to occupants. Finally, special systems such as Heat Recovery and deploying of interior shading are presented as well. Once simulated, results for each scenario were compared for each building, as well as to the baseline as shown in the following section.

The results where then plotted and visually integrated into the dashboard design. Each building's simulated past, present and future energy usage was compared using records from their own historical data, compared in the form of potential future energy reductions between scenarios, or to other buildings utilizing EUI results to avoid total energy misconceptions.

\section{Results}

The results are divided into energy consumption reductions due to single-action and multiple action scenarios. Each simulation outcome is compared to the baseline and shown as a percentage reduction in the energy use category being investigated, as well as in total energy use. The results assume that users would be motivated to change their behaviour upon learning the impact of their action on energy consumption. There may be some adaptation required in terms of comfort. For example, changing cooling set points will require users to be accepting to an action that favours energy efficiency over their preferred choices for cooling set points.

\section{Single-action scenarios}

A total of nine scenarios were simulated for the three buildings in the community, each corresponding to a different energy consumption cluster group. The following section is a breakdown of the different energy load types and their respective energy savings as compared to the baseline.

\section{- Cooling}

The simulation of a total of five cooling-oriented energy saving scenarios demonstrated that with the focus on reducing cooling energy loads, total energy consumption could result in reductions up to $18.7 \%$. For all three buildings the lowering set points scenario (S1) had the greatest impact, reducing total cooling energy by $31.4 \%$ for the low, $27.38 \%$ for medium and $41.28 \%$ for the high cluster building. Shading (S6) resulted in cooling energy reductions of $31.27 \%, 22.12 \%$ and $32.01 \%$ respectively, with reductions of $18.64 \%, 13.32 \%$ and $14.35 \%$ for total energy use. The next greater cooling impact was through S9 (lights off during the day) and S8 (natural ventilation), which reduced total energy consumption by $7.98 \%$, $7.07 \%, 11.39 \%$ and totals of $7.07 \%, 2.43 \%, 8.34 \%$, respectively. Two of the scenarios expected to have a significant impact on cooling loads, (S2 and S3) demonstrated no change or minimal impact on energy reduction.

\section{- Lighting}

Results demonstrated that scenarios five (Reduction in Power Density) - was 50\% lighting energy reduction for all types, nine (Lights off during the day) - 38.9\%, 38,9\% and $37.34 \%$ for high, mid and low, respectively, and four (Lower illuminance target, dimming control) $-33.01 \%$, $29.57 \%$ and $24.88 \%$ as well. Results from the simulations also demonstrated that by utilizing lighting-energy saving strategies, total energy could be reduced up to $11.39 \%$.

\section{- Equipment}

Only one of all simulated scenarios presented changes in equipment energy consumption values. The simulations demonstrated that by reducing Equipment Power Density from $3 \mathrm{~W} / \mathrm{m}^{2}$ to $1 \mathrm{~W} / \mathrm{m}^{2}$, lighting energy use would show reductions of $66.6 \%$ in all analysed buildings, consequently reducing total energy consumption by $24.45 \%, 18.71 \%$ and $28.46 \%$ for low, medium and highenergy analysis buildings respectively.

\section{- Total energy}

Our research demonstrated that individual actions such as the reduction of Equipment Power Density, reduction of set points and the addition of interior shading respectively 
were the best options to reduce total energy consumption within the community. Figure 4 shows the respective changes in total EUI values for all simulated one-action scenarios.
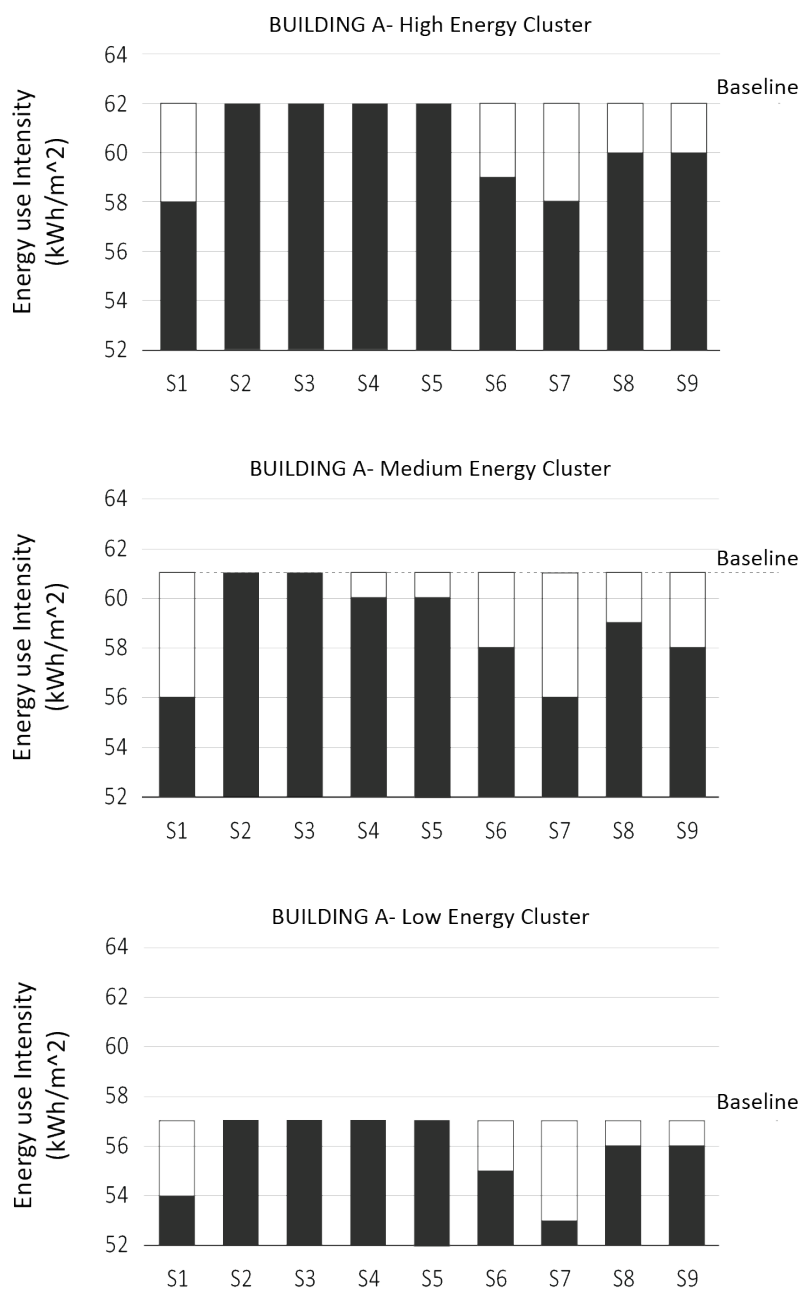

Figure 4: Change in EUI for three tested cases.

\section{Combined Scenarios}

Results from simulated single-action scenarios demonstrated potential total energy savings for the community of $28 \%$ approximately. However, further research was conducted for combined scenarios to investigate the possibility for additional savings in energy consumption. A total of four combined scenarios were simulated, from which one focused on the further reduction of cooling loads, another on equipment, then lighting, and finally, a combination of the most-energy efficient actions presented in the previous section. Results were as follows:

\section{- C-I: Set-Points + Shading}

The simulation of the first combined scenario focused on investigating further reducing cooling energy loads. Results demonstrated a reduction of $34.47 \%, 33.5 \%$ and $28.54 \%$ of total energy consumption for low, medium and high energy use buildings respectively. With the respective reduction of $7 \mathrm{kWh} / \mathrm{m}^{\wedge} 2,7 \mathrm{kWh} / \mathrm{m}^{\wedge} 2$ and $5 \mathrm{kWh} / \mathrm{m}^{\wedge} 2$ in total EUI values.
- C-II: Lighting+ Equipment Power Density

Results for the second combination demonstrated that by lowering the power density of both lighting and equipment, total energy consumption would decrease by a respective $28.32 \%, 11.7 \%$ and $40.3 \%$ for low, medium and high usage buildings, as well as a reduction of $5 \mathrm{kWh} / \mathrm{m}^{\wedge} 2,6 \mathrm{kWh} / \mathrm{m}^{\wedge} 2$ and $5 \mathrm{kWh} / \mathrm{m}^{\wedge} 2$ on total EUI values respectively.

\section{- C-III: Lights off + Lighting Power Density}

The simulation of a lighting-focused combined scenario with lower Lighting Power Density and a low-energy lighting usage schedule, which assumes no lighting usage during daylight hours, resulted in significant reductions of $13.6 \%, 11.2 \%$ and $8.77 \%$ in total energy usage for low, medium and high usage buildings respectively. And reductions of $3 \mathrm{kWh} / \mathrm{m}^{\wedge} 2,4 \mathrm{kWh} / \mathrm{m}^{\wedge} 2$ and $1 \mathrm{kWh} / \mathrm{m}^{\wedge} 2$ in total EUI.

\section{- C-IV: Low Set-Points + Shading +} Equipment/Lighting Power Density

Results from the simulation of an all-combination scenario which included low set-points, interior shading and low equipment and lighting power density inputs demonstrated significant reductions in total energy savings as follows: $62 \%$ reduction for low, $51.25 \%$ for medium and $67.46 \%$ for high energy analysis buildings, with a respective reduction of $13 \mathrm{kWh} / \mathrm{m}^{\wedge} 2,12 \mathrm{kWh} / \mathrm{m}^{\wedge} 2$ and $10 \mathrm{kWh} / \mathrm{m}^{\wedge} 2$ in total EUI values.

\section{Discussion - Performance Dashboards}

The results demonstrated through simulation that significant reductions in energy consumption of residential buildings can be achieved through the selection and combination of specific eco-friendly curtailment actions. The speed by which future energy usage can be deduced and visualized using UBEM tools implies that simulation-based Eco-feedback is an achievable step towards the successful development of an engaging and tailored interactive Eco-feedback platform.

The findings suggest that through the visualization of validated simulated data, users should be able to a) receive accurate information for their current energy use and also compare their previous historical energy data, $b$ ) visualize future scenarios for their energy usage and the respective energy savings, c) make selections for desired actions and receive educational feedback about impacts on their energy usage, while comparing them to past, present or future measured and simulated energy data. We speculate that with the attentive representation and communication of simulated data, such benefits as well as the enthusiastic interaction and long-term user engagement with their energy consumption could be pursued. Utilizing findings from literature research as well as information from previous research, schemes of a dashboard were developed and designed to display measured and simulated data for the community. Figure 5 shows the designed schematic Eco-feedback dashboard.

There are various dashboards in use as reviewed in the literature, but a very limited number have the capability to project different future scenarios, and inform residents 
about which actions would have the greatest impact on their energy use, electricity bill and carbon footprint. Tips for energy reduction that include recommendations such as reducing overnight plug loads may not be as effective as the utilization of shading, changing setpoint settings and eliminating daytime lighting. This approach to feedback can be tailored for residential users, rather than providing generic and unvalidated recommendations.

\section{Long-term User Engagement}

There is a need for users to better understand how their behaviours impact their energy bill, and lack of education and engagement has become a limitation for most energy visualization platforms. Challenges related to understanding and translating numerical data into meaningful information, or to being able to permanently engage with a non-visual scientific interface are considered as the primary reasons why dashboard model for other communities in various climate regions, as it is adaptable to various levels of data granularity with regards to various energy end uses and engagement behaviour.

Further development of dashboard schemes for tailored Eco-feedback could provide comparisons between neighbours, and links to social media platforms. Previous literature has demonstrated that goal-setting comparative actions, as well as social recognition can serve as a successful incentive for the implementation of ecological behaviours. Critically, future work should also include testing dashboard designs with community stakeholders to provide feedback on the potentials, as well as barriers, to utilizing such Eco-feedback technologies for sustained engagement.

\section{Conclusion}

This paper presented workflows that employ urban-scale

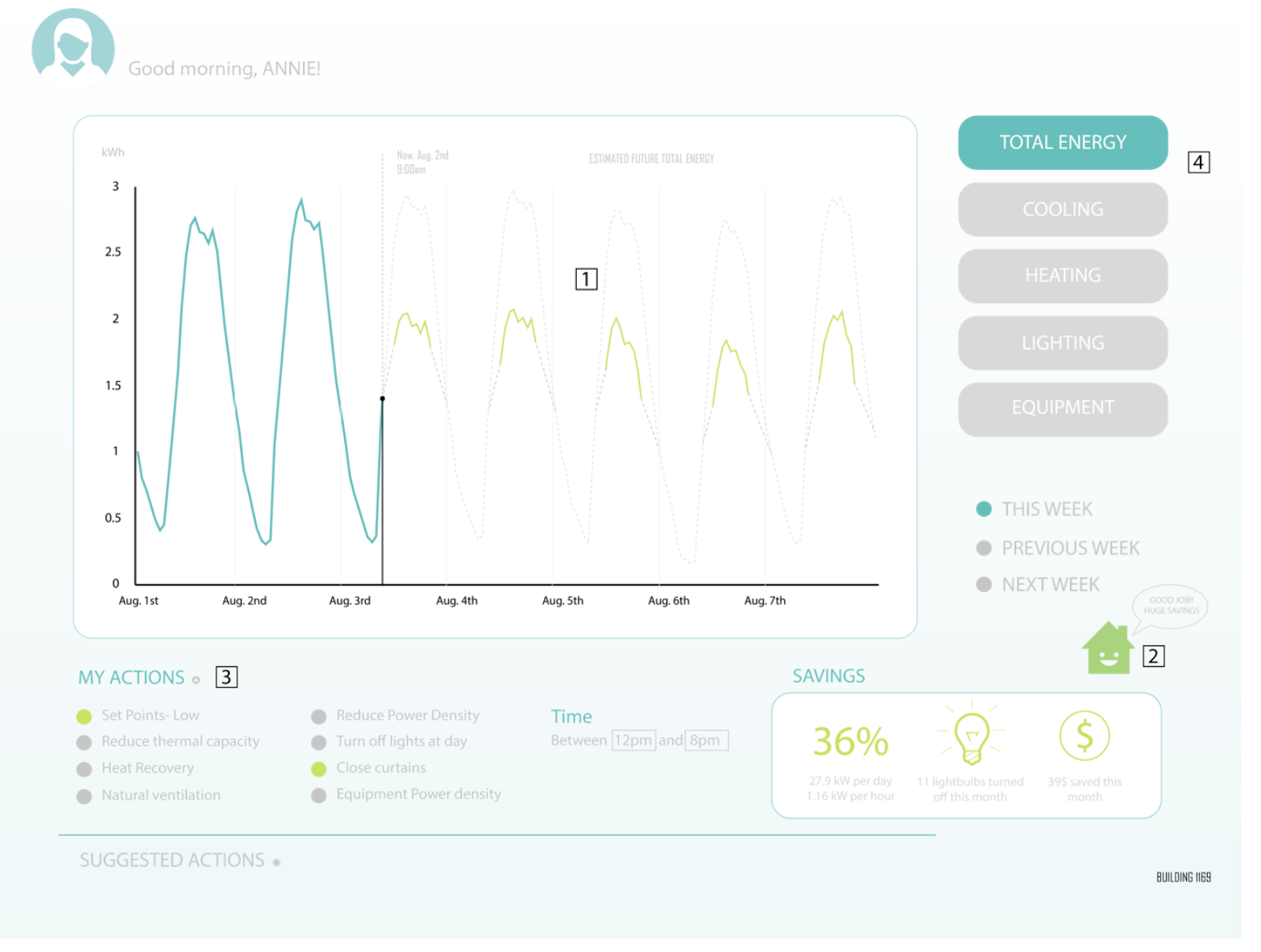

Figure 5: A performance dashboard mock-up that demonstrates: (1) Comparisons between present and historical data. (2) Energy awareness icons designed to give feedback to the user. (3) A selectable actions toolbar linked with simulation results, so that users can select and visualize different future options for their energy consumption. (4) Different tabs for Cooling, Heating, Lighting, Equipment and Total Energy.

interfaces are not considered a priority in the users' energy-conscious maintenance tasks.

The design of the presented dashboard attempts to provide the user with understandable, customizable feedback in both the form of numerical and visually engaging information. This approach is therefore presented as a simulation-based predictions of future residential energy use to provide real-time tailored display feedback of various energy behaviour and design alteration scenarios. Utilizing a cause-effect approach, the user would visualize and compare past and present home energy usage, learn about recommended strategies to reduce 
consumption, make individual decisions and changes towards more sustainable behaviours, and visualize their long-term impact, as well as compare progress and savings within their community. Through calibrated simulation of design-case scenarios, using a UBEM, the dashboard is expected to serve as a stakeholder education tool to identify sustainable actions in achieving energy efficiency. The presented visualizations are intended to serve as a schematic reference for future work. The primary objective for the simulation and design of the dashboard interface is to provide a framework for future advancement in the development of interactive userengagement platforms, as well as to providing solutions to the study limitations, such as the lack of real-time simulation workflows. Future work should implement such sustainable urban community Eco-feedback through simulation-enabled performance dashboards and test user engagement with various stakeholders, and identify means of continuous engagements to monitor the active reduction of energy use and consequent carbon emissions in residential buildings.

\section{Acknowledgement}

This publication is based on work funded in part by the National Science Foundation (NSF), under the Smart and Connected Communities program grant 1737550 , and the Syracuse Center of Excellence Faculty Fellows program. The authors would like to thank the Pecan Street Institute for providing access to measured energy data for the Mueller community in Austin, Texas. The authors are also grateful for the work that student Yu Qian Wang provided to support this manuscript.

\section{References}

Attari, S. Z., DeKay, M. L., Davidson, C. I., \& De Bruin, W. B. (2010). Public perceptions of energy consumption. (W. C. Clark, Ed.) Proceedings of the National Academy of Sciences of the United States of America, 107(37), 16054-16059.

Crawley, D. B., Lawrie, L. K., Winkelmann, F. C., Buhl, W. F., Huang, Y. J., Pedersen, C. O., ... and Glazer, J. (2001). EnergyPlus: creating a new-generation building energy simulation program. Energy and buildings, 33(4), 319-331.

Bouveyron, C., Côme, E. and Jacques, J., (2015). The discriminative functional mixture model for a comparative analysis of bike sharing systems. The Annals of Applied Statistics, 9(4), pp. 1726-1760.

Constanza, E., Ramchurn, S. D., \& Jennings, N. R. (2012). Understanding Domestic Energy Consumption. UbiComp '12 (pp. 216-225). Pittsburgh: ACM.

Fleming, J. R. (1998). Historical Perspectives on Climate Change. Oxford: Oxford University Press.
Gardner, G. T., and Stern, P. C. (2008). The Short List: The Most Effective Actions U.S. Environment: Science and Policy for Sustainable, 50(5), 12-25.

Hensen, J. (1992). Energy Simulation in Building Design. Wroclaw interuniversity research seminar, (pp. 101-112). Eindhoven.

Horn, M. S., Davis, P., Hubbard, A. K., Keifert, D., Atrash Leong, Z., \& Olson, I. C. (2011). Learning Sustainability: Families, Learning, and NextGeneration Eco-Feedback Technology. Interaction Design and Children Conference, (pp. 161-165). Ann Arbor.

Karlin, Beth, Ford, Rebecca, and Cassandra Squiers. (2014). "Energy Feedback Technology: A Review and Taxonomy of Products and Platforms." Energy Efficiency 7 (3): 377-399.

Pachauri, R. K., Allen, M. R., Barros, V. R., Broome, J., Cramer, W., Christ, R., ... and Dubash, N. K. (2014). Climate change 2014: synthesis report. Contribution of Working Groups I, II and III to the fifth assessment report of the Intergovernmental Panel on Climate Change. IPCC.

Petersen, S. and Svendsen, S. (2010). Method and simulation program informed decisions in the early stages. Energy and Buildings, 42, 1113-1119.

Pierce, J., Odom, W., and Blevis, E. (2008). Energy Aware Dwelling: A Critical Survey of Interaction. Computer-Human Interaction: Designing for Habitus and Habitat (pp. 1-8). Cairns: CHISIG.

Pittock, B. A. (2005). Climate Change, Turning Up the Heat. London: Routledge.

El Kontar, R., and Rakha, T. (2018). Profiling Occupancy Patterns to Calibrate Urban Building Energy Models (UBEMs) Using Measured Data Clustering. Technology| Architecture + Design, 2(2), 206-217.

Reinhart, C. F., Dogan, T., Jakubiec, J. A., Rakha, T., \& Sang, A. (2013, August). Umi-an urban simulation environment for building energy use, daylighting and walkability. In 13th Conference of International Building Performance Simulation Association, Chambery, France.

Schwarz, G. (1978). Estimating the dimension of a model. The annals of statistics, 6(2), 461-464.

Yamaguchi, Y., Shimoda, Y., and Mizuno, M. (2007). Proposal of a modeling approach considering urban form of evaluation of city level energy management. Energy and Buildings, 39, 580-592. 